\title{
The inhibition of Notch signaling ameliorates the motor functional deficits and astrocytic abnormality of in vitro and in vivo spinal muscular atrophy models
}

\author{
Kazuki Ohuchi ${ }^{1,2}$, Michinori Funato ${ }^{2}$, Shiori Ando ${ }^{1,2}$, Satoshi Inagaki ${ }^{1,2}$, Chizuru Kawase ${ }^{2}$, \\ Junko Seki ${ }^{2}$, Shinsuke Nakamura ${ }^{1}$, Masamitsu Shimazawa ${ }^{1}$, Hideo Kaneko ${ }^{2}$, Hideaki Hara ${ }^{1}$ \\ ${ }^{1}$ Molecular Pharmacology, Gifu Pharmaceutical University, Japan, ${ }^{2}$ Department of Clinical Research, National \\ Hospital Organization, Nagara Medical Center, Japan
}

Background

Spinal muscular atrophy (SMA) is an autosomal recessive neuromuscular disorder characterized by the degeneration of spinal motor neurons and muscle atrophy. This disease is mainly caused by the low level of the survival motor neuron (SMN) protein that mainly leads to spinal motor neuron degeneration. Previous reports showed that SMN-depleted astrocyte was abnormally increased in the spinal cord. However, no researcher groups elucidated the mechanism of SMNdepleted astrocytic abnormality. Our purpose is to identify the signaling associated with SMN-depleted astrocytic abnormality and to propose the candidate therapy tool by modulating the signaling.

Methods

In this study, we used SMN $\Delta 7$ mice as in vivo SMA model, which was widely used as SMA model mice. The astrocytic abnormality in the spinal cord of SMN $\Delta 7$ mice at postnatal day (PND) 11 was evaluated by immunohistochemistry using astrocytic markers such as glial fibrillary acidic protein (GFAP) and S100. The expression of Notch intracellular domain (NICD) and phosphorylated signal transducers and activator of transcription 3 (p-STAT3) was measured using Westernblot analysis at PND5 and 11 of SMN $\Delta 7$ mice. The effect of a Notch inhibitor against the GFAP positive astrocytes derived from SMA patient's iPS cell was evaluated by immunostaining using anti-GFAP antibody. Furthermore, the effect of a Notch inhibitor at $1.4 \mathrm{ng} /$ body (i.c.v) in $\operatorname{SMN} \Delta 7$ mice was tested by survival time, motor performance including righting reflex and negative geotaxis.

Results

First, GFAP-positive astrocytes were abnormally increased in the gray matter of spinal cord and S100-positive astrocytes were also increased in specific to central canal of spinal cords. Second, Notch signaling and astrogenic signal were activated in the spinal cord of SMN $\Delta 7$ mice at in early (PND5) and late stages (PND11) of SMN $\Delta 7$ mice. Finally, the pharmacological inhibition of Notch signaling improved the motor functional deficits of SMN $\Delta 7$ mice.

Conclusion

These results suggest GFAP-positive astrocytes were increased via the activation of Notch signaling and the pharmacological inhibition of the Notch signaling is the one of the therapeutic targets in the SMA pathology. 